\title{
Neural plasticity and consciousness
}

\section{Mads Jensen* and Morten Overgaard}

Cognitive Neuroscience Research Unit, Hammel Neurorehabilitation and Research Center, Aarhus University Hospital, Aarhus, Denmark

*Correspondence: mads@cnru.dk

Neural plasticity has, despite a growing interest in neuroscience over the last decades, received little interest in consciousness research. This might be because neural plasticity has no clear and strict definition; it is used to describe the ability of neuronal tissue to adjust activities and physical characteristics in order to adapt to changes in the environment or changes in their use - in relation to, e.g., behavioral patterns, cognition, or metabolic demands. The term traces back in history and was discussed by William James (1910-1942) and Ramón y Cajal (1852-1934) who provided evidence to argue for local plasticity in structure and function in otherwise fixed neural circuits.

Neural plasticity covers a number of different types of changes in brain tissue or organization, which arguably can be described at a high or a low level and at different time scales. At "lower" levels of description, a very simple example of plasticity is facilitation, i.e., the increase in amplitude of a postsynaptic potential after rapid activations from other, presynaptic neurons. Long-term potentiation (LTP), being the persistency of the postsynaptic potential after the facilitating stimulus has subsided, may lead to increased postsynaptic activities for days. Other examples of "low level processes" that could be mentioned are, changes in individual neurons or small circuits involve synaptogenesis (the creation of individual or groups of synapses to build connections between neurons) and neuronal migration (where neurons "travel" to become part of circuits distant from their site of "birth").

At a "higher" level of description, reorganizations related to changes in cognition may be investigated. In cognitive neuroscience, an impressive amount of evidence suggests a functional localization of mental phenomena in the brain, so that different cognitive and emotional functions are associated with specific networks or regions of the brain (e.g., Frackowiak et al., 2003). Functional reorganization involves a change in how, for example, a specific cognitive function is realized in the brain. Such a change will in some instances be the result of neural repair mechanisms following an injury to the brain. In such cases, functional reorganizations will typically be considered cases of functional recovery where a wide range of functions (e.g., language) seemingly are realized in different brain regions after injury to regions previously associated with the function (Overgaard and Mogensen, 2011).

The concept of consciousness is even more complicated and has even more different interpretations than the concept of neural plasticity. We, like several others in this field of research, take the concept to mean subjective or phenomenal experience. One primary question very frequently asked among those interested in consciousness is which specific neural regions or networks that are necessary and/or sufficient for a person to be conscious. This is referred to as the neural correlate of consciousness (NCC).

The NCC is traditionally defined as "the minimal set of neuronal events and mechanisms jointly sufficient for a specific conscious percept" (Koch, 2004, p. 16) or "A neural system N is an NCC if the state of $\mathrm{N}$ correlates directly with states of consciousness" (Chalmers, 2000, p. 18). At a first look, such a statement claims nothing more than a neural system $\mathrm{N}$ at a given point in time correlates directly with states of consciousness. However, we assume the claim is intended to be stronger than that, namely that an NCC in some fundamental aspects is the same over time and between individuals. In other words, the NCC for a conscious experience $\mathrm{X}$ is not just the NCC at $a$ time - it is the NCC at any time.

Research on neural plasticity can be taken to imply that a simple one-to-one mapping between brain structure and mental phenomenon might not be an optimal strategy in the attempt to reveal the neural underpinnings of consciousness. If it proves to be the case that "one and the same" mental state may be related to several different physical processes, an explanation should rather look for common factors among different sufficient "realisers" of consciousness than it would merely identify brain regions.

A classical interpretation of the relation between conscious experience (qualia) and brain processes has been that qualia are determined by the brain activity, such that if the brain activity had been different then the conscious experience would have been different as well. Such an interpretation, in the light of research on neural plasticity, seems very difficult. Rather, research on neural plasticity seems more in support of a weaker view on localization, arguing that mechanisms or cognitive structure is "realized in" brain activity or brain structure, which is not restricted to a certain localization.

The concept of neural plasticity captures a diverse and complex range of phenomena, as argued above, with different levels of explanation. The integration of theories about neural plasticity at different levels and theories of consciousness is therefore complex, carrying important implications for the plausibility of any conception of the relationship of consciousness and brain processes. With this introductory paper, we hope to start an otherwise neglected debate, unfolded in the Special Topic "Consciousness and Neural Plasticity."

\section{ACKNOWLEDGMENTS}

Mads Jensen was supported by a grant from The Danish Council for Independent Research | Humanities. Morten Overgaard was supported by a starting grant from The European Research Council.

\section{REFERENCES}

Chalmers, D. J. (2000). "What is a neural correlate of consciousness?" in Neural Correlates of Consciousness, ed. T. Metzinger (Cambridge: MIT Press), 17-39.

Frackowiak, R., Friston, K. J., Frith, C., Donal, R., Price, C. J., Zeki, S., Ashburner, J., and Penny, W. D. (2003). Human Brain Function, 2nd Edn. San Diego: Academic Press. 
Koch, C. (2004). The Quest for Consciousness: A Neurobiological Approach. Englewood, CO: Roberts and Co.

Overgaard, M., and Mogensen, J. (2011). A framework for the study of multiple realizations: the importance of levels of analysis. Front. Psychol. 2:79. doi: 10.3389/ fpsyg.2011.00079
Received: 20 June 2011; accepted: 27 July 2011; published online: 01 September 2011.

Citation: Jensen $M$ and Overgaard M (2011) Neural plasticity and consciousness. Front. Psychology 2:191. doi: 10.3389/fpsyg.2011.00191

This article was submitted to Frontiers in Consciousness Research, a specialty of Frontiers in Psychology.
Copyright (C) 2011 Jensen and Overgaard. This is an open-access article subject to a non-exclusive license between the authors and Frontiers Media SA, which permits use, distribution and reproduction in other forums, provided the original authors and source are credited and other Frontiers conditions are complied with. 